\title{
Employing RHIC and LHC data to determine the transverse momentum dependent gluon density in a proton
}

\author{
N. A. Abdulov, ${ }^{1}$ H. Jung, ${ }^{2}$ A. V. Lipatov, ${ }^{3,4}$ G. I. Lykasov, ${ }^{4}$ and M. A. Malyshev ${ }^{3}$ \\ ${ }^{1}$ Faculty of Physics, Lomonosov Moscow State University, 119991 Moscow, Russia \\ ${ }^{2}$ Deutsches Elektronen-Synchrotron, 22603 Hamburg, Germany \\ ${ }^{3}$ Skobeltsyn Institute of Nuclear Physics, Lomonosov Moscow State University, 119991 Moscow, Russia \\ ${ }^{4}$ Joint Institute for Nuclear Research, 141980 Dubna, Moscow Region, Russia
}

(Received 22 June 2018; published 11 September 2018)

\begin{abstract}
Transverse momentum dependent (TMD) parton distributions in a proton are important in high-energy physics from both theoretical and phenomenological points of view. Using the latest RHIC and LHC data on the inclusive soft hadron production in $p p$ and $A A$ collisions at small transverse momenta, we determine the parameters of the initial TMD gluon density derived in the framework of a quark-gluon string model at the low scale $\mu_{0} \sim 1-2 \mathrm{GeV}$ and refine its large- $x$ behavior using the LHC data on the $t \bar{t}$ production at $\sqrt{s}=13 \mathrm{TeV}$. Then, we apply the Catani-Ciafaloni-Fiorani-Marchesini evolution equation to extend the obtained TMD gluon density to the whole kinematical region. In addition, the complementary TMD valence and sea quark distributions are generated. The latter are evaluated in the approximation where the gluon-to-quark splitting occurs at the last evolution step using the TMD gluon-to-quark splitting function. Several phenomenological applications of the proposed TMD quark and gluon densities to the LHC processes are discussed.
\end{abstract}

DOI: 10.1103/PhysRevD.98.054010

\section{INTRODUCTION}

In recent years, an understanding has been obtained that a theoretical description of a number of processes at high energies and large momentum transfer containing multiple hard scales requires unintegrated, or transverse momentum dependent (TMD) parton density functions [1], which encode nonperturbative information on a proton structure, including transverse momentum and polarization degrees of freedom. The TMD parton densities are related to the physical cross sections and other observables measured in the collider experiments via TMD factorization theorems in quantum chromodynamics (QCD). At present, the CollinsSoper-Sterman factorization approach designed for semiinclusive processes with a finite and nonzero ratio between the hard scale and total energy [2], and the high-energy factorization [3] (or the $k_{T}$-factorization [4]) approach valid in the limit of a fixed hard scale and high energy, are developed. The factorization theorems provide the necessary framework to separate hard partonic physics described with a perturbative QCD expansion from soft hadronic physics and allow one to determine the TMD parton distributions from collider data.

Published by the American Physical Society under the terms of the Creative Commons Attribution 4.0 International license. Further distribution of this work must maintain attribution to the author(s) and the published article's title, journal citation, and DOI. Funded by SCOAP.
In the high-energy factorization, the production cross sections at low transverse momenta are governed by the nonperturbative input to the TMD parton density functions. The latter being used as an initial condition for the subsequent QCD evolution could play an important role in phenomenological applications [5-8]. As it was shown [9-11], its influence on the description of the experimental data can be significant. At present, several approaches to determine the TMD gluon density in a proton (or rather, its initial parameters) are known in the literature. In the Kimber-Martin-Ryskin scheme developed at leading order (LO) [12] and next-to-leading order (NLO) [13], the TMD quark and gluon densities are derived from the conventional parton distribution functions. At low $k_{T} \leq \mu_{0} \sim 1 \mathrm{GeV}$, they are defined from a simple normalization condition. Recently, the TMD quark and gluon densities in a proton were determined [14] from fits to precision measurements of deep inelastic scattering cross sections at HERA and evolved by the Dokshitzer-Gribov-Lipatov-AltarelliParisi (DGLAP) evolution [15] with NLO splitting functions using the parton branching method $[16,17]$. In a more complicated approach [18] based on the Catani-CiafaloniFiorani-Marchesini (CCFM) gluon evolution equation [19], the parameters of initial TMD gluon distribution were fitted from the precision HERA data on proton structure function $F_{2}\left(x, Q^{2}\right)$ in the range $x<5 \times 10^{-3}, Q^{2}>5 \mathrm{GeV}^{2}$, and $F_{2}^{c}\left(x, Q^{2}\right)$ at $Q^{2}>2.5 \mathrm{GeV}^{2}$ assuming the Gaussianlike dependence on the intrinsic gluon transverse 
momentum $k_{T}$ at $k_{T} \leq \mu_{0} \sim 2 \mathrm{GeV}$. In our previous papers [9-11], the initial TMD gluon density was derived in the framework of the soft quark-gluon string model [20-22] by taking into account gluon saturation effects at low $Q^{2}$. The essential parameters were obtained from the best description of the inclusive spectra of hadrons produced in $p p$ collisions at LHC energies in the midrapidity region at low transverse momenta $p_{T} \leq 4.5 \mathrm{GeV}$. Being used with the CCFM evolution, the predictions based on the proposed TMD gluon density describe well the HERA measurements of the proton structure functions $F_{2}^{c}\left(x, Q^{2}\right), F_{2}^{b}\left(x, Q^{2}\right)$, and $F_{L}\left(x, Q^{2}\right)$. Thereby, the connection between soft LHC processes and small- $x$ physics at HERA in a wide kinematical region was established. An important advantage of the approaches $[10,11,18]$ is that one can rather easily take into account a large piece of higher-order corrections, namely, part of $\mathrm{NLO}+\mathrm{NNLO}+\cdots$ terms containing leading $\log 1 / x$ enhancement of cross sections due to real initial state parton emissions absorbed into the CCFM evolution $^{1}$ (see [24] for more information).

In the present paper, we continue our previous studies [9-11] and test the parameters of the initial TMD gluon density [9-11] using the recent NA61 [25], LHC [26$31]$, and RHIC [32,33] data for soft hadron production in $p p$ and $A A$ collisions obtained in a wide energy range. Moreover, we refine its large- $x$ behavior using the latest LHC data on the inclusive top quark pair production at $\sqrt{s}=13 \mathrm{TeV}$ [34]. Following Refs. [10,11], we extend the updated TMD gluon distribution to the whole range of the longitudinal momentum fraction $x$, transverse momentum $\mathbf{k}_{T}^{2}$, and hard scale $\mu^{2}$ numerically using the UPDFEVOLV package [35], which is the CCFM evolution code for TMD parton densities. In our opinion, the CCFM equation is the most suitable tool for our study since it smoothly interpolates between the small- $x$ Balitsky-Fadin-Kuraev-Lipatov [36] (BFKL) gluon dynamics and the conventional DGLAP one. We supply the obtained TMD gluon density with the corresponding TMD valence and sea quark distributions calculated in the approximation, where the sea quarks occur in the last gluon splitting. Finally, we discuss several phenomenological applications of the proposed TMD parton densities to hard LHC processes sensitive to the quark and gluon content of the proton.

The paper is organized as follows. In Sec. II, we describe how we determine the initial gluon density from the LHC data and discuss its subsequent QCD evolution. In Sec. III, we illustrate the use of the obtained TMD gluon density at the LHC. We give conclusions in Sec. IV.

\footnotetext{
${ }^{1}$ At present, most of the proposed TMD parton distributions in a proton are collected in the TMDLIB package [23], which is a C++ library providing a framework and an interface to the different parametrizations.
}

\section{NONPERTURBATIVE TMD GLUON INPUT AND EVOLUTION}

The determination of the parameters of the initial TMD gluon density in a proton can, in fact, be split into the two almost independent pieces referring to the regions of small and large $x$. We consider first the small- $x$ region and start from the simple analytical expression for the starting TMD gluon distribution function at some fixed scale $\mu_{0} \sim 1-2 \mathrm{GeV}$. It can be presented in the form [11]

$f_{g}^{(0)}\left(x, \mathbf{k}_{T}^{2}, \mu_{0}^{2}\right)=\tilde{f}_{g}^{(0)}\left(x, \mathbf{k}_{T}^{2}, \mu_{0}^{2}\right)+\lambda\left(x, \mathbf{k}_{T}^{2}, \mu_{0}^{2}\right) f_{g}\left(x, \mathbf{k}_{T}^{2}\right)$,

where $x$ and $\mathbf{k}_{T}$ are the proton longitudinal momentum fraction and two-dimensional gluon transverse momentum, respectively. The first term $\tilde{f}_{g}^{(0)}\left(x, \mathbf{k}_{T}^{2}, \mu_{0}^{2}\right)$ was calculated [9] within the soft QCD model and reads

$$
\begin{aligned}
\tilde{f}_{g}^{(0)}\left(x, \mathbf{k}_{T}^{2}, \mu_{0}^{2}\right)= & c_{0} c_{1}(1-x)^{b}\left[R_{0}^{2}(x) \mathbf{k}_{T}^{2}+c_{2}\left(R_{0}^{2}(x) \mathbf{k}_{T}^{2}\right)^{a / 2}\right] \\
& \times \exp \left(-R_{0}(x)\left|\mathbf{k}_{T}\right|-d\left[R_{0}^{2}(x) \mathbf{k}_{T}^{2}\right]^{3 / 2}\right),
\end{aligned}
$$

where $R_{0}^{2}(x)=\left(x / x_{0}\right)^{\lambda} / \mu_{0}^{2}$ and $c_{0}=3 \sigma_{0} / 4 \pi^{2} \alpha_{s}$. The parameters $\sigma_{0}=29.12 \mathrm{mb}, \lambda=0.22, x_{0}=4.21 \times 10^{-5}$, and $\alpha_{s}=0.2$ come from the Golec-Biernat-Wüsthoff (GBW) saturation model [37], while the other parameters $a, b, c_{1}, c_{2}$, and $d$ were fitted from LHC data on inclusive spectra of charged hadrons. The numerical values of these parameters, details of the calculations, and the relation between the TMD gluon density and the inclusive hadron spectra are given in our previous papers [9-11]. The gluon density $\tilde{f}_{g}^{(0)}\left(x, \mathbf{k}_{T}^{2}, \mu_{0}^{2}\right)$ differs from the one obtained in the GBW model at $\left|\mathbf{k}_{T}\right|<1 \mathrm{GeV}$ and coincides with the GBW gluon at larger $\left|\mathbf{k}_{T}\right|>1.5 \mathrm{GeV}$ [11]. The second term $f_{g}\left(x, \mathbf{k}_{T}^{2}\right)$ represents the analytical solution [38] of the linear BFKL equation at low $x$ weighted with a matching function $\lambda\left(x, \mathbf{k}_{T}^{2}, \mu_{0}^{2}\right)$ :

$$
\begin{gathered}
f_{g}\left(x, \mathbf{k}_{T}^{2}\right)=\alpha_{s}^{2} x^{-\Delta} t^{-1 / 2} \frac{1}{v} \exp \left[-\frac{\pi \ln ^{2} v}{t}\right], \\
\lambda\left(x, \mathbf{k}_{T}^{2}, \mu_{0}^{2}\right)=c_{0}\left(\frac{x}{x_{0}}\right)^{0.81} \exp \left[-k_{0}^{2} \frac{R_{0}(x)}{\left|\mathbf{k}_{T}\right|}\right],
\end{gathered}
$$

where $t=14 \alpha_{s} N_{c} \zeta(3) \ln (1 / x), \quad \Delta=4 \alpha_{s} N_{c} \ln 2 / \pi, \quad v=$ $\left|\mathbf{k}_{T}\right| / \Lambda_{\mathrm{QCD}}$, and $k_{0}=1 \mathrm{GeV}$. This term allows one to describe LHC measurements of inclusive charged hadrons up to $p_{T} \leq 4.5 \mathrm{GeV}$ [9]. It is important that the contribution from $f_{g}\left(x, \mathbf{k}_{T}^{2}\right)$ is only nonzero at $\left|\mathbf{k}_{T}\right| \ll \Lambda_{\mathrm{QCD}}(1 / x)^{\delta}$ with $\delta=\alpha_{s} N_{c}$, resulting in an average generated gluon transverse momentum of $\left\langle\left|\mathbf{k}_{T}\right|\right\rangle \sim 1.9 \mathrm{GeV}$. The latter value is close to the nonperturbative $\mathrm{QCD}$ regime that allows one to 

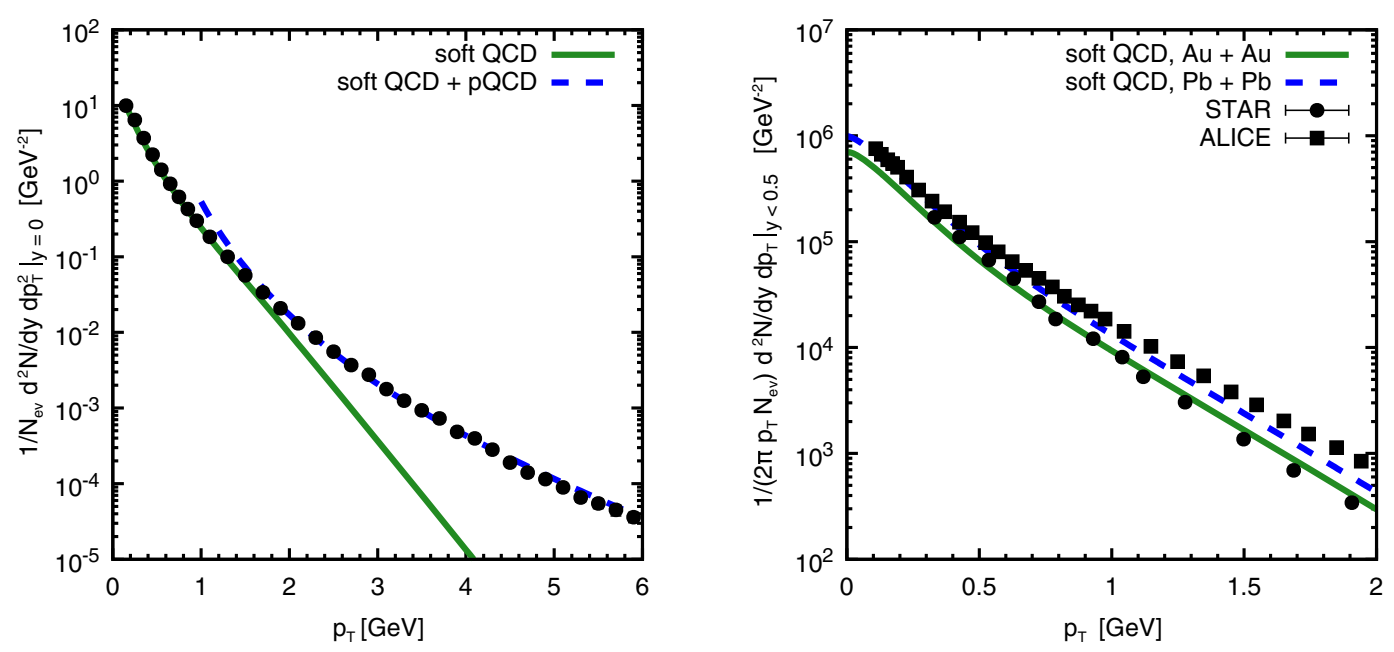

FIG. 1. Left panel: The inclusive cross section of charge hadrons produced in $p p$ collisions as a function of their transverse momentum at $\sqrt{s}=7 \mathrm{TeV}$. The experimental data are from CMS and ATLAS [26,27]. Right panel: Pion transverse mass spectra in $\mathrm{Au}+\mathrm{Au}$ at $\sqrt{s}=200 \mathrm{GeV}$ and $\mathrm{Pb}+\mathrm{Pb}$ collisions at $\sqrt{s}=2.76 \mathrm{TeV}$. The experimental data are from STAR [32,33] and ALICE [28-31].

treat the TMD gluon density above as a starting one for the CCFM evolution.

Previously, the phenomenological parameters $a, b, c_{1}$, $c_{2}$, and $d$ in (1)-(4) were determined in the small- $x$ region only, where $x \sim 1 \times 10^{-4}-1 \times 10^{-5}$ (see [9-11]). The fit was based on NA61 data on inclusive cross sections of $\pi^{-}$ meson production in $p p$ collisions at initial momenta 31 and $158 \mathrm{GeV}$ [25] and on CMS [26] and ATLAS [27] data on inclusive hadron production in $p p$ collisions at the LHC. In the present paper, we tested all these parameters using the experimental data on the pion transverse mass distribution in $\mathrm{Au}+\mathrm{Au}$ and $\mathrm{Pb}+\mathrm{Pb}$ collisions taken by the STAR Collaboration at the RHIC [32,33] and the ALICE Collaboration at the LHC [28-31]. The details of the calculations of hadron production cross sections in $A A$ collisions are given in [39].

Next, we note that determination of the parameters of the TMD gluon density in the small- $x$ region could result only in significant theoretical uncertainties of the predictions and/or poor description of the data at moderate and large- $x$ values. Therefore, in the present paper, we refine some of these parameters essential in the large- $x$ region using recent experimental data on inclusive $t \bar{t}$ production taken by the CMS Collaboration at $\sqrt{s}=$ $13 \mathrm{TeV}$ [34]. These data refer to $x \sim 2 m_{t} / \sqrt{s} \sim 3 \times 10^{-2}$ (with a top mass $m_{t} \sim 170 \mathrm{GeV}$ ) and are reported at the parton level in the full phase space, allowing us to avoid the numerical simulation of top quark decays. To calculate the $t \bar{t}$ production cross sections in the $k_{T}$-factorization approach, we follow our previous consideration [40]. We find that $b=10$ and $d=0.4$ are more preferable to describe the distributions on the rapidity and transverse momentum of top quark pairs. The latter leads, in addition, to a different value of overall normalization $n=0.27$ in (1), which was determined using the CMS data on inclusive $b$-jet production.

Then, we test the other parameters from a fit on RHIC $[32,33]$ and LHC data [28-31] on soft hadron production in $p p$ and $A A$ collisions. The hadron production in $p p$ collisions at $p_{T}<2 \mathrm{GeV}$ is fitted with $\chi^{2} /$ n.d.f. $=0.9$. The resulting cross sections are compared with the data in Fig. 1, where the soft QCD predictions include both gluon and quark contributions. The perturbative QCD corrections calculated $[41,42]$ at LO are divergent at low transverse momenta ${ }^{2}$ (not shown for $A A$ collisions). We would like to note here that the approach [39] with the above-determined parameters of the TMD gluon density is able to describe the experimental data in a wide energy range. Concerning the large- $x$ region, the achieved description of the CMS data [34] on the top pair production is illustrated in Fig. 2, where the transverse momentum and rapidity distributions of the top quarks are shown as an example. For the reader's convenience, we collected all the parameters of (1)-(4) in Table I.

Next, we extend the obtained TMD gluon density (1)-(4) to a higher scale $\mu^{2}$ using the CCFM evolution equation. This equation resums large logarithms $\alpha_{s}^{n} \ln ^{n} 1 / x$ and $\alpha_{s}^{n} \ln ^{n} 1 /(1-x)$ and, therefore, is valid at both small and large $x$ (see, e.g., [24] for more information). In the leading logarithmic approximation, ${ }^{3}$ the CCFM equation with respect to the evolution scale $\mu^{2}$ can be written as

\footnotetext{
${ }^{2}$ The kinematical region $p_{T} \sim 1.8-2.2 \mathrm{GeV}$ can be treated as the matching region of the soft QCD and pQCD calculations.

${ }^{3}$ The next-to-leading logarithmic corrections for the CCFM equation are still unknown. However, as it was argued [43], amending the leading logarithmic evolution with kinematical constraint $[44,45]$ leads to reasonable QCD predictions, although still formally only to leading logarithmic accuracy (see, also, [24]).
} 

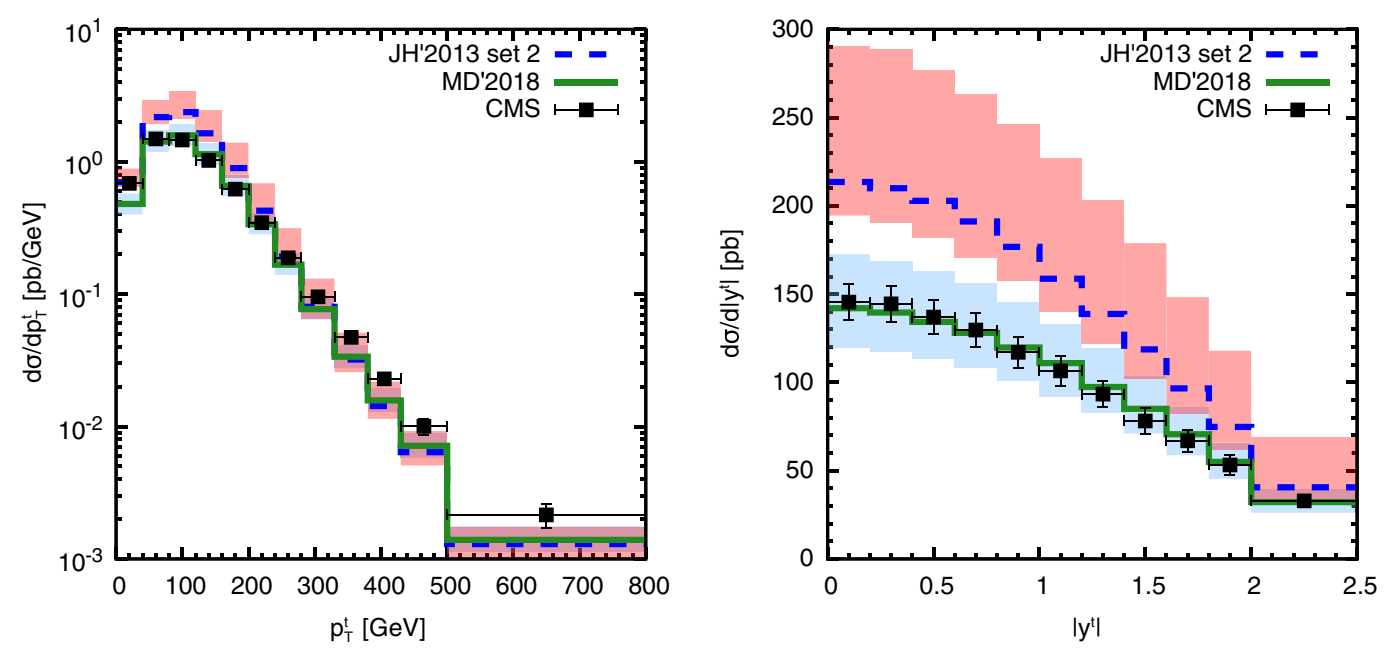

FIG. 2. The transverse momentum and rapidity distributions of inclusive $t \bar{t}$ production in $p p$ collisions at $\sqrt{s}=13$ TeV. The green (solid) and blue (dashed) curves correspond to the predictions obtained using the MD'2018 and JH'2013 set 2 gluons, respectively. The shaded bands represent their scale uncertainties. The experimental data are from CMS [34].

$$
f_{g}\left(x, \mathbf{k}_{T}^{2}, \mu^{2}\right)=f_{g}^{(0)}\left(x, \mathbf{k}_{T}^{2}, \mu_{0}^{2}\right) \Delta_{s}\left(\mu^{2}, \mu_{0}^{2}\right)+\int \frac{d z}{z} \int \frac{d q^{2}}{q^{2}} \theta(\mu-z q) \Delta_{s}\left(\mu^{2}, z^{2} q^{2}\right) P_{g g}\left(z, q^{2}, \mathbf{k}_{T}^{2}\right) f_{g}\left(x / z, \mathbf{k}_{T}^{\prime 2}, q^{2}\right),
$$

where $\mathbf{k}_{T}^{\prime}=\mathbf{q}(1-z)+\mathbf{k}_{T}$. The exact analytical expressions for the Sudakov form factor $\Delta_{s}\left(p^{2}, q^{2}\right)$ and gluon splitting functions $P_{g g}\left(z, q^{2}, \mathbf{k}_{T}^{2}\right)$ can be found, e.g., in [35]. The CCFM equation with the starting TMD gluon density $f_{g}^{(0)}\left(x, \mathbf{k}_{T}^{2}, \mu_{0}^{2}\right)$ given by (1)-(4) was solved numerically using the UPDFEVOLV package [35]. As it was done earlier [11], to produce the TMD valence and sea quark distributions, we apply the approach of Ref. [46]. So, the TMD sea quark density was calculated in the approximation where the sea quarks occur in the last gluon splitting:

$$
\begin{aligned}
& f_{q}^{(s)}\left(x, \mathbf{k}_{T}^{2}, \mu^{2}\right) \\
& =\int_{x}^{1} \frac{d z}{z} \int d \mathbf{q}_{T}^{2} \frac{1}{\Delta^{2}} \frac{\alpha_{s}}{2 \pi} P_{q g}\left(z, \mathbf{q}_{T}^{2}, \Delta^{2}\right) f_{g}\left(x / z, \mathbf{q}_{T}^{2}, \bar{\mu}^{2}\right),
\end{aligned}
$$

where $z$ is the fraction of the gluon light-cone momentum carried by the quark and $\Delta=\mathbf{k}_{T}-z \mathbf{q}_{T}$. The hard scale $\bar{\mu}^{2}$ was defined [47] from the angular ordering condition which is natural from the CCFM point of view: $\bar{\mu}^{2}=\boldsymbol{\Delta}^{2} /(1-z)^{2}+$ $\mathbf{q}_{T}^{2} /(1-z)$. The off-shell gluon-to-quark splitting function $P_{q g}\left(z, \mathbf{q}_{T}^{2}, \Delta^{2}\right)$ was calculated in [48].

TABLE I. Numerical values of the parameters of the TMD gluon density (1)-(4). All other parameters, namely, $x_{0}, \sigma_{0}, \lambda$, and $\alpha_{s}$ are unchanged.

\begin{tabular}{lcccccc}
\hline \hline Parameter & $a$ & $b$ & $c_{1}$ & $c_{2}$ & $d$ & $\mu_{0} / \mathrm{GeV}$ \\
\hline Fitted value & 0.3 & 10.0 & 0.3295 & 2.3 & 0.4 & 2.2 \\
\hline \hline
\end{tabular}

The gluon density $f_{g}\left(x, \mathbf{k}_{T}^{2}, \mu^{2}\right)$ obtained according to (1)-(5) labeled below as Moscow-Dubna 2018, or MD'2018, is shown in Fig. 3 versus the longitudinal momentum fraction $x$ and transverse momentum $\mathbf{k}_{T}$ at different evolution scales. Additionally, we plot the TMD gluon distribution [18] (namely, the JH'2013 set 2), which is widely discussed in the literature and commonly used in applications. One can observe some difference in the absolute normalization and shape between both TMD gluon distributions. In particular, the $\mathbf{k}_{T}$ tail of the MD'2018 density function is the contribution due to the solution of the linear BFKL equation, as was described above. Actually, it was needed to improve the description of the LHC data on charge hadron production in $p p$ collisions at $\sqrt{s}=7 \mathrm{TeV}$ and $2.5<$ $p_{T}<4 \mathrm{GeV}$ (see [9] for more details). Below, we will consider the phenomenological consequences for several LHC processes. ${ }^{4}$

\section{PHENOMENOLOGICAL APPLICATIONS}

We are now in a position to apply the MD'2018 gluon density to several hard processes studied at hadron colliders. We use the $k_{T}$-factorization approach, where the production cross section of any process under consideration (say, in $p p$ collisions) can be written as

\footnotetext{
${ }^{4}$ The MD'2018 gluon density will be implemented in the forthcoming release of the TMDLIB package.
} 

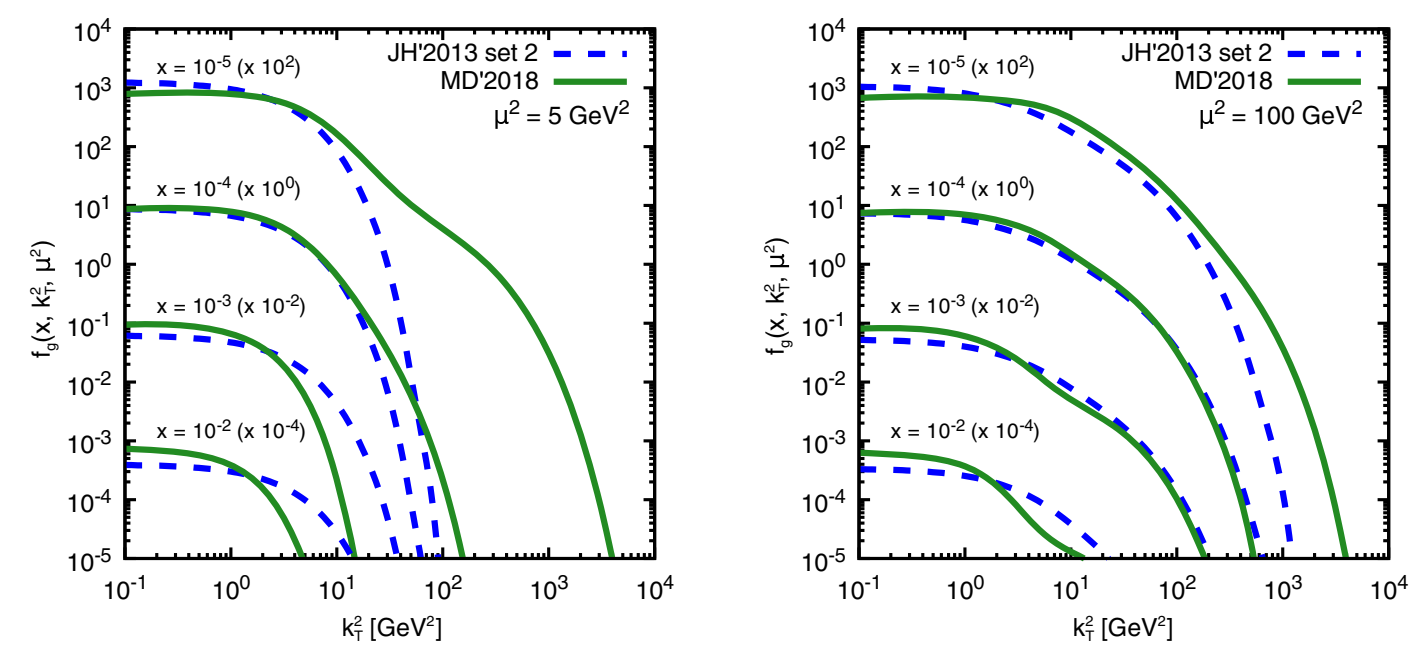

FIG. 3. The TMD gluon densities in the proton calculated as a function of the gluon transverse momentum $\mathbf{k}_{T}^{2}$ at different longitudinal momentum fraction $x$ and $\mu^{2}$ values. The green (solid) and blue (dashed) curves correspond to the MD'2018 and JH'2013 set 2 gluon density functions, respectively.

$$
\sigma=\sum_{i, j} \int d x_{1} d x_{2} \int d \mathbf{k}_{1 T}^{2} d \mathbf{k}_{2 T}^{2} f_{i / h_{1}}\left(x_{1}, \mathbf{k}_{1 T}^{2}, \mu^{2}\right) f_{j / h_{2}}\left(x_{2}, \mathbf{k}_{2 T}^{2}, \mu^{2}\right) \times d \hat{\sigma}_{i j}\left(x_{1}, x_{2}, \mathbf{k}_{1 T}^{2}, \mathbf{k}_{2 T}^{2}, \mu^{2}\right),
$$

where $i, j$ denote the partons $(g, q$, or $\bar{q}$ ) inside colliding particles $h_{1,2}$ (protons or heavy ions), and $\hat{\sigma}_{i j}\left(x_{1}, x_{2}, \mathbf{k}_{1 T}^{2}, \mathbf{k}_{2 T}^{2}, \mu^{2}\right)$ is the corresponding off-shell (depending on the transverse momenta of incoming particles) partonic cross section. Everywhere below, the multidimensional integration was performed by the Monte Carlo technique, using the routine veGAS [49].

\section{A. Proton structure functions $F_{2}^{c}$ and $F_{2}^{b}$}

It is well known that the basic information on the proton structure can be extracted from deep inelastic $e p$ scattering. Its differential cross section can be presented in the simple form:

$\frac{d^{2} \sigma}{d x d y}=\frac{2 \pi \alpha^{2}}{x Q^{4}}\left[\left(1-y+\frac{y^{2}}{2}\right) F_{2}\left(x, Q^{2}\right)-\frac{y^{2}}{2} F_{L}\left(x, Q^{2}\right)\right]$,

where $F_{2}\left(x, Q^{2}\right)$ and $F_{L}\left(x, Q^{2}\right)$ are the proton transverse and longitudinal structure functions, and $x$ and $y$ are the usual Bjorken scaling variables. In the present paper, we concentrate on the charm and beauty contributions to $F_{2}\left(x, Q^{2}\right)$. The latter are described through perturbative generation of charm and beauty quarks and, therefore, directly related with the gluon content of the proton. Our evaluation below is based on the formulas [50]. Numerically, we apply the pole mass $m_{c}=1.7 \mathrm{GeV}$ and $m_{b}=4.8 \mathrm{GeV}$ and strictly follow our previous consideration [50] in all other aspects.
Our results are shown in Figs. 4 and 5 in comparison with the latest ZEUS [51] and $\mathrm{H} 1$ data $[52,53]$. The green and grey curves correspond to the predictions obtained with the MD'2018 and JH'2013 set 2 gluon densities, whereas the shaded bands represent the estimations of the scale uncertainties of these calculations. We find that the MD'2018 predictions for $F_{2}^{c}\left(x, Q^{2}\right)$ and $F_{2}^{b}\left(x, Q^{2}\right)$ are in reasonable agreement with the HERA data in a wide region of $x$ and $Q^{2}$, both in overall normalization and shape. It slightly overshoots the JH'2013 set 2 predictions at small $Q^{2}$ and low $x \leq 10^{-4}$. At larger $Q^{2}$ and moderate and/or large $x \geq 10^{-2}$, the JH'2013 set 2 gluon density function tends to overestimate the HERA data on the structure function $F_{2}^{b}\left(x, Q^{2}\right)$, which is due to the determination of input parameters of this gluon density at small $x$ only [18]. Therefore, the influence of the shape and other parameters of the initial nonperturbative gluon distribution on the description of the collider data is significant for a wide region of $x$ and $Q^{2}$ [9-11]. The MD'2018 gluon density, where all these parameters are verified by the description of LHC data, leads to a better agreement with the HERA data, which confirms the link between soft processes at the LHC and low- $x$ physics at HERA pointed out earlier [9-11]. Note that to estimate the scale uncertainties of the JH'2013 set 2 calculations, the method proposed in [18] was used. So, to evaluate the latter, we used the JH'2013 set $2+$ and JH'2013 set 2- sets instead of the default one JH'2013 set 2 . These two sets represent a variation of the renormalization scale used in the off-shell production amplitude: the JH'2013 set $2+$ stands for a variation of $2 \mu_{R}$, while 


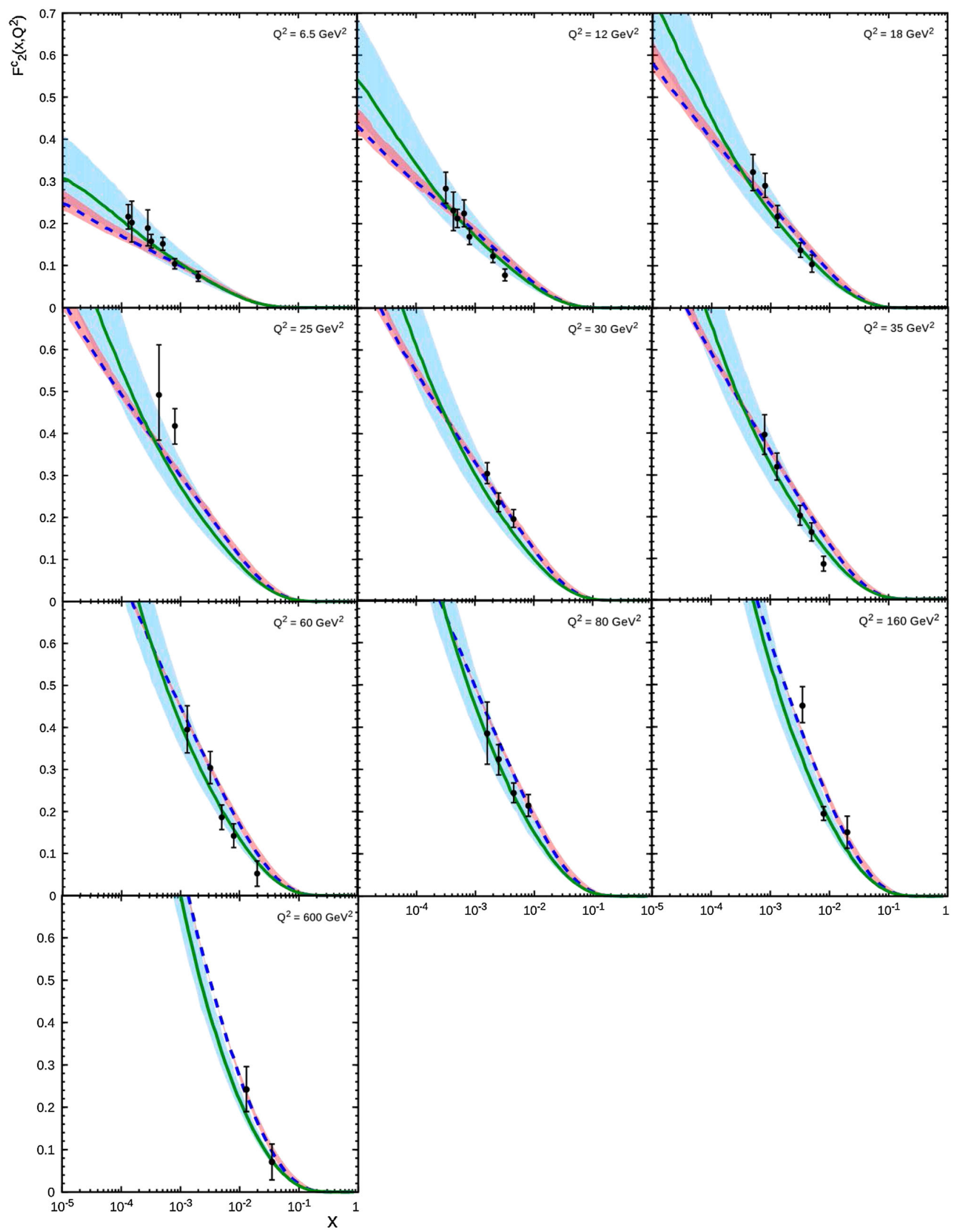

FIG. 4. The charm contribution to the proton structure function $F_{2}\left(x, Q^{2}\right)$ as a function of $x$ calculated at different $Q^{2}$. Notation of the histograms is the same as in Fig. 2. The experimental data are from ZEUS [51] and H1 [52]. 


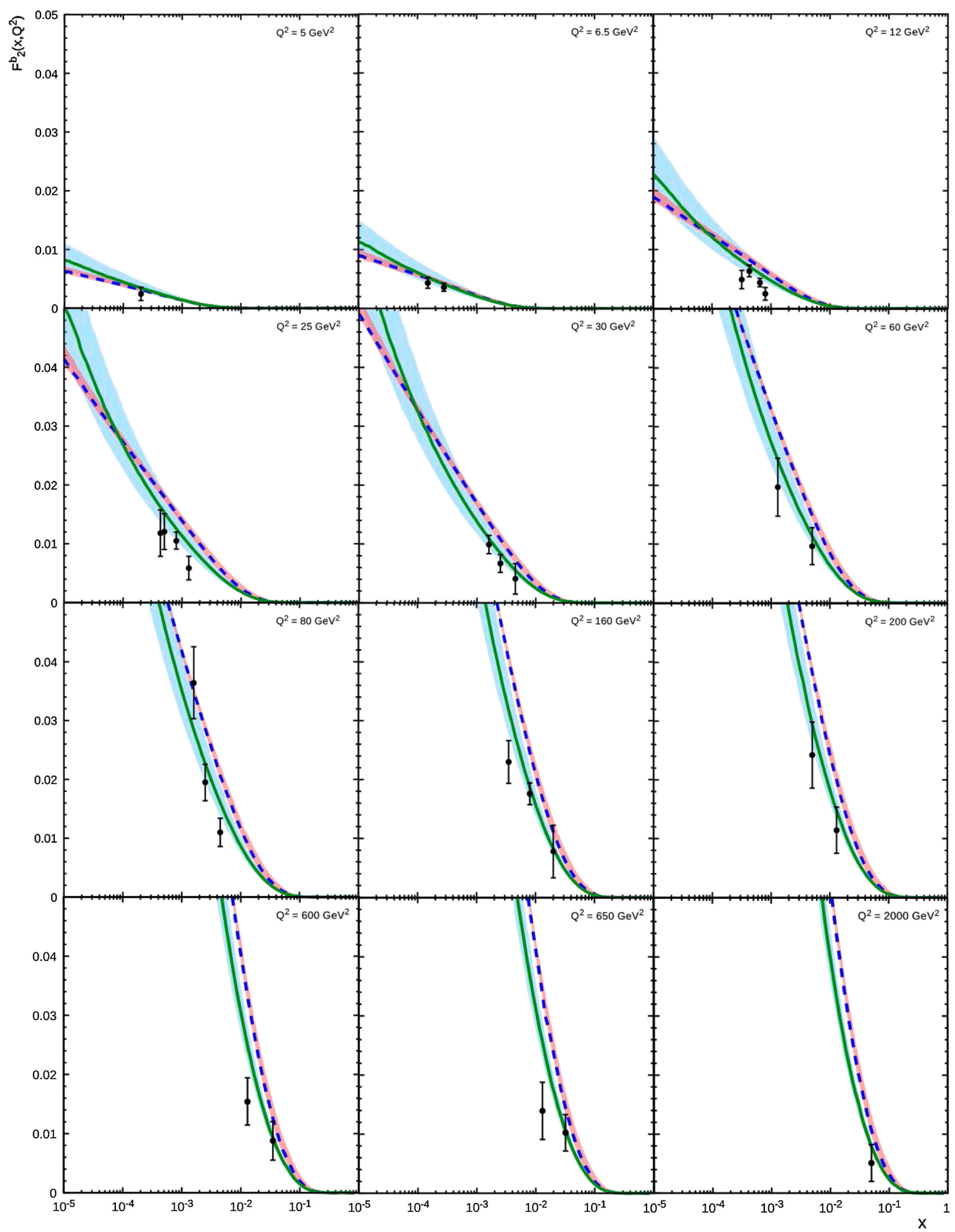

FIG. 5. The beauty contribution to the proton structure function $F_{2}\left(x, Q^{2}\right)$ as a function of $x$ calculated at different $Q^{2}$. Notation of the histograms is the same as in Fig. 2. The experimental data are from ZEUS [51] and H1 [53]. 
JH'2013 set $2-$ reflects $\mu_{R} / 2$. This method leads to somewhat reduced uncertainty bands in comparison with the MD'2018 predictions.

\section{B. Single top production at the LHC}

Recently, the CMS and ATLAS Collaborations have measured the differential cross sections of single top production (in the $t$ channel) at the LHC as a function of the transverse momenta and rapidity of the top quark and top antiquark at $\sqrt{s}=8 \mathrm{TeV}[54,55]$. Such measurements are known to be very useful for constraining parton densities in a proton [56,57]. To calculate the total and differential production cross sections, we employ the four-flavor scheme so that the leading contribution comes from the $2 \rightarrow 3$ off-shell (reggeized) quark-gluon interaction subprocess:

$$
q^{*}\left(k_{1}\right)+g^{*}\left(k_{2}\right) \rightarrow q^{\prime}\left(p_{1}\right)+\bar{b}\left(p_{2}\right)+t(p),
$$

where the four-momenta of all particles are indicated in parentheses. The main contribution to the amplitude (8) comes from the diagram, which corresponds to initial gluon splitting to a $b \bar{b}$ pair with subsequent exchange of the $W$ boson between the $b$ and the light quark. The latter reads

$$
\begin{aligned}
\mathcal{A}= & -g \frac{e^{2}}{8 \sin ^{2} \theta_{W}} V_{q q^{\prime}} V_{t b} \bar{u}_{s_{1}}\left(p_{1}\right) \Gamma_{(+)}^{\mu}\left(k_{1},-p_{1}\right)\left(1-\gamma^{5}\right) u_{s_{2}}\left(x_{1} l_{1}\right) \\
& \times \bar{u}_{s_{3}}(p) \gamma_{\mu}\left(1-\gamma^{5}\right) \frac{\hat{k}_{2}-\hat{p}_{2}+m_{b}}{\left(k_{2}-p_{2}\right)^{2}-m_{b}^{2}} \hat{\epsilon}\left(k_{2}\right) v_{s_{4}}\left(p_{2}\right) t^{a} \frac{1}{\left(p_{1}-k_{1}\right)^{2}-m_{W}^{2}+i m_{W} \Gamma_{W}},
\end{aligned}
$$

where $g$ and $e$ are the strong and electric charges, respectively, $\theta_{W}$ is the weak Weinberg mixing angle, $V_{q_{a} q_{b}}$ are the Cabibbo-Kobayashi-Maskawa matrix elements, $m_{b}$ and $m_{W}$ are the $b$-quark and $W$-boson masses, $a$ is the eightfold color index, and $\Gamma_{W}$ is the $W$-boson full decay width. The effective vertex $\Gamma_{(+)}^{\mu}(k, q)$ that ensures gauge invariance of the amplitude (9) despite the off-shell initial partons can be written as $[58,59]$

$$
\Gamma_{(+)}^{\mu}(k, q)=\gamma^{\mu}-\hat{k} \frac{l_{1}^{\mu}}{l_{1} \cdot q}
$$

where $l_{1}$ is the proton four-momentum $\left(k_{1}=x_{1} l_{1}+k_{1 T}\right.$ and $k_{2}=x_{2} l_{2}+k_{2 T}$ ). The polarization sum for the off-shell gluon is taken in the BFKL form $[3,4]$ :

$$
\sum \epsilon^{\mu}(k) \epsilon^{\nu}(k)=\frac{k_{T}^{\mu} k_{T}^{\nu}}{k_{T}^{2}} .
$$

In all other aspects, the calculation is straightforward and follows standard Feynman rules. The evaluation of traces was performed using the algebraic manipulation system FORM.

Having calculated the squared amplitude (9), one can evaluate the total and differential cross sections of single top production according to the TMD factorization formula (7). Numerically, we took $m_{W}=80.4 \mathrm{GeV}$ and $\Gamma_{W}=2.1 \mathrm{GeV}$. The light quarks were kept massless, while for heavy quarks we took $m_{b}=4.75 \mathrm{GeV}$ and $m_{t}=175 \mathrm{GeV}$. The weak mixing angle was chosen to correspond to $\sin ^{2} \theta_{W}=0.23$ [60]. As the renormalization $\mu_{R}$ and factorization $\mu_{F}$ scales, we choose the largest mass parameter in our calculation, the top transverse mass. ${ }^{5}$

\footnotetext{
${ }^{5}$ The different choices of hard scales in the single top production are discussed in $[57,61]$.
}

The results of our calculations for single top quark production in the $t$ channel are presented in Figs. 6-8 in comparison with the CMS and ATLAS data [54,55]. These data correspond to the absolute and normalized differential cross sections on parton level as functions of top quark transverse momentum and rapidity. Studying the latter could lead to a more stringent comparison between data and theory due to reduced experimental (mainly systematic) and theoretical (scale) uncertainties. We find that both the MD'2018 and JH'2013 set 2 gluon densities predict almost identical normalized cross sections, which agree with the CMS and ATLAS measurement quite well. However, the MD'2018 density results in a little smaller total cross section than the JH'2013 set 2 one that leads to somewhat better description of the data. Thus, the calculations endorse the usage of the MD'2018 gluon density for the evaluation of cross sections of processes with quite large- $x$ values involved. Note that the size of scale uncertainties of MD'2018 and JH'2013 set 2 calculations are rather close to each other in the kinematical region probed.

\section{Inclusive Higgs boson production at the LHC}

Very recently, the CMS and ATLAS Collaborations have reported measurements [62-65] of the total and differential cross sections of inclusive Higgs boson production at $\sqrt{s}=$ $13 \mathrm{TeV}$ obtained in different Higgs decay channels. These measurements can be used to investigate the gluon dynamics in a proton since the dominant mechanism of inclusive Higgs production at the LHC is gluon-gluon fusion [66-69]. Here, to calculate the total and differential cross sections of Higgs boson production, we strictly follow our previous consideration [70]. The latter is based on the off-shell amplitude of the gluon-gluon fusion subprocess $g^{*} g^{*} \rightarrow H$ calculated using the effective Lagrangian [71,72] for the 

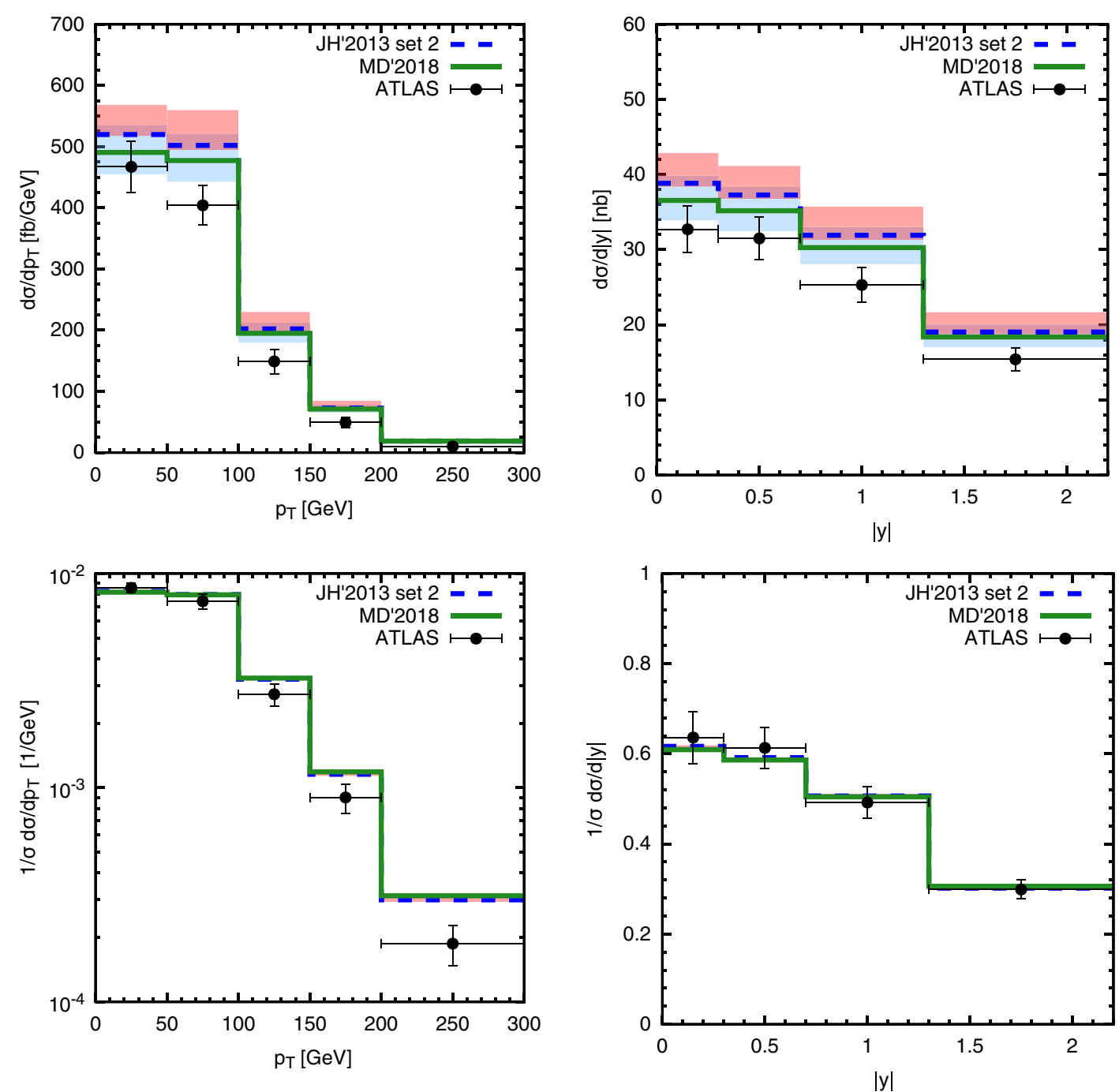

FIG. 6. The differential cross sections of inclusive $t$-channel single top production at $\sqrt{s}=8 \mathrm{TeV}$ as functions of top quark transverse momentum and rapidity. Notation of the histograms is the same as in Fig. 2. The experimental data are from ATLAS [55].

Higgs coupling to gluons and extended recently to the subsequent $H \rightarrow \gamma \gamma, H \rightarrow W^{+} W^{-} \rightarrow e^{ \pm} \mu^{\mp} \nu \bar{\nu}$ [73], and $H \rightarrow Z Z^{*} \rightarrow 4 l$ decays $[73,74]$. The details of the calculations are explained in [73]. Below, we present the numerical results obtained with the MD'2018 and JH'2013 set 2 gluon densities for $H \rightarrow \gamma \gamma$ and $H \rightarrow Z Z^{*} \rightarrow 4 l$ decay modes.

The CMS and ATLAS measurements refer to a restricted part of the phase space (fiducial phase space) defined to match the experimental acceptance in terms of the photon kinematics and event selection. In the CMS analysis [62], two isolated photons originating from the Higgs boson decays are required to have pseudorapidities $\left|\eta^{\gamma}\right|<2.5$. Photons with largest and next-to-largest transverse momentum $p_{T}^{\gamma}$ (so-called leading and subleading photons) must satisfy the conditions of $p_{T}^{\gamma} / m^{\gamma \gamma}>1 / 3$ and $p_{T}^{\gamma} / m^{\gamma \gamma}>1 / 4$, respectively, where the diphoton mass $m^{\gamma \gamma}$ is required to be $100<m^{\gamma \gamma}<180 \mathrm{GeV}$. In the ATLAS measurement [64], both decay photons must have pseudorapidities $\left|\eta^{\gamma}\right|<2.37$ with the leading (subleading) photon satisfying $p_{T}^{\gamma} / \mathrm{m}^{\gamma \gamma}>$ 0.35 (0.25), while invariant mass $m^{\gamma \gamma}$ is required to be $105<m^{\gamma \gamma}<160 \mathrm{GeV}$. In the $H \rightarrow Z Z^{*} \rightarrow 4 l$ decay channel, only events with a four-lepton invariant mass $118<$ $m_{4 l}<129 \mathrm{GeV}$ are kept by the ATLAS Collaboration [65], and each lepton (electron or muon) must satisfy the transverse momentum cut $p_{T}>6 \mathrm{GeV}$ and be in the pseudorapidity range $|\eta|<2.47$. The highest- $p_{T}$ lepton in the quadruplet must have $p_{T}>20 \mathrm{GeV}$, and the second (third) lepton in $p_{T}$ order must satisfy $p_{T}>15(10) \mathrm{GeV}$. These leptons are required to be separated from each other by $\Delta R=\sqrt{(\Delta \eta)^{2}+(\Delta \phi)^{2}}>0.1(0.2)$ when having the same (different) lepton flavors. The invariant mass $m_{12}$ of the lepton pair closest to the $Z$-boson mass (leading pair) is required to be $50<m_{12}<106 \mathrm{GeV}$. The subleading pair is chosen as the remaining lepton pair with invariant mass $m_{34}$ closest to the $Z$-boson mass and satisfying the 

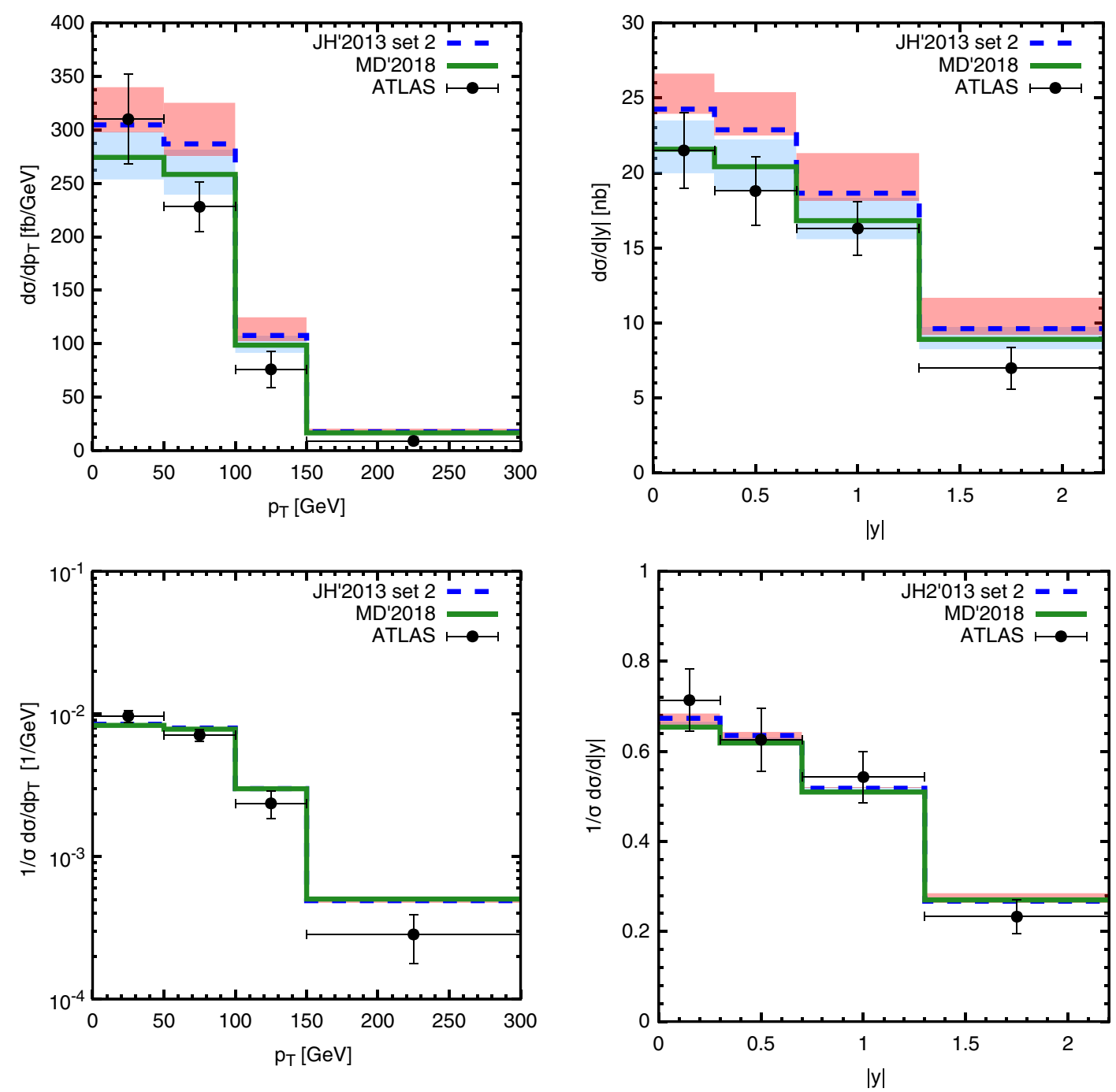

FIG. 7. The differential cross sections of inclusive $t$-channel single antitop production at $\sqrt{s}=8 \mathrm{TeV}$ as functions of $\bar{t}$ quark transverse momentum and rapidity. Notation of the histograms is the same as in Fig. 2. The experimental data are from ATLAS [55].

requirement $12<m_{34}<115 \mathrm{GeV}$. The $\mathrm{CMS}$ measurement [63] requires at least four leptons in the event with at least one lepton having $p_{T}>20 \mathrm{GeV}$, another lepton having $p_{T}>10 \mathrm{GeV}$, and the remaining ones having $p_{T}>7$ and $5 \mathrm{GeV}$, respectively. All leptons must have the pseudorapidity $|\eta|<2.4$, the leading pair invariant mass $m_{12}$ must be $40<m_{12}<120 \mathrm{GeV}$, and the subleading one should be $12<m_{34}<120 \mathrm{GeV}$. Finally, the fourlepton invariant mass $m_{4 l}$ must satisfy the $105<m_{4 l}<$ $140 \mathrm{GeV}$ cut.

The results of our calculations are shown in Figs. 9 and 10 in comparison with the latest LHC data [62-65]. In the $H \rightarrow \gamma \gamma$ decay channel, we calculated the distributions on the diphoton pair transverse momentum $p_{T}^{\gamma \gamma}$, absolute value of the rapidity $\left|y^{\gamma \gamma}\right|$, photon helicity angle $\left|\cos \theta^{*}\right|$ (in the Collins-Soper frame), and azimuthal angle difference $\Delta \phi^{\gamma \gamma}$ between the produced photons. In the $H \rightarrow Z Z^{*} \rightarrow 4 l$ decay channel, we calculated distributions on the Higgs transverse momentum $p_{T}^{H}$, rapidity $\left|y^{H}\right|$, invariant mass of the subleading lepton pair $m_{34}$, and cosine of the leading lepton pair decay angle $\left|\cos \theta^{*}\right|$ in the four-lepton rest frame with respect to the beam axis. We find that both the MD'2018 and JH'2013 set 2 predictions reasonably agree with the data within the uncertainties for all considered kinematical observables, although the MD'2018 results lie a bit below the JH'2013 set 2 ones. Some tendency to underestimate the LHC data at large transverse momenta could be explained by the missing contributions from the weak boson fusion $\left(W^{+} W^{-} \rightarrow H\right.$ and $\left.Z Z \rightarrow H\right)$ and/or associated $H Z$ or $H W^{ \pm}$production [75], which become important at high $p_{T}$ and are not taken into account in our present consideration. The measured rapidity, $\left|\cos \theta^{*}\right|$, and $m_{34}$ distributions are well reproduced by our calculations. As one can see, despite the fact that both the MD'2018 and JH'2013 set 2 gluon distributions agree with the available data, the inclusive Higgs boson production at the LHC is 

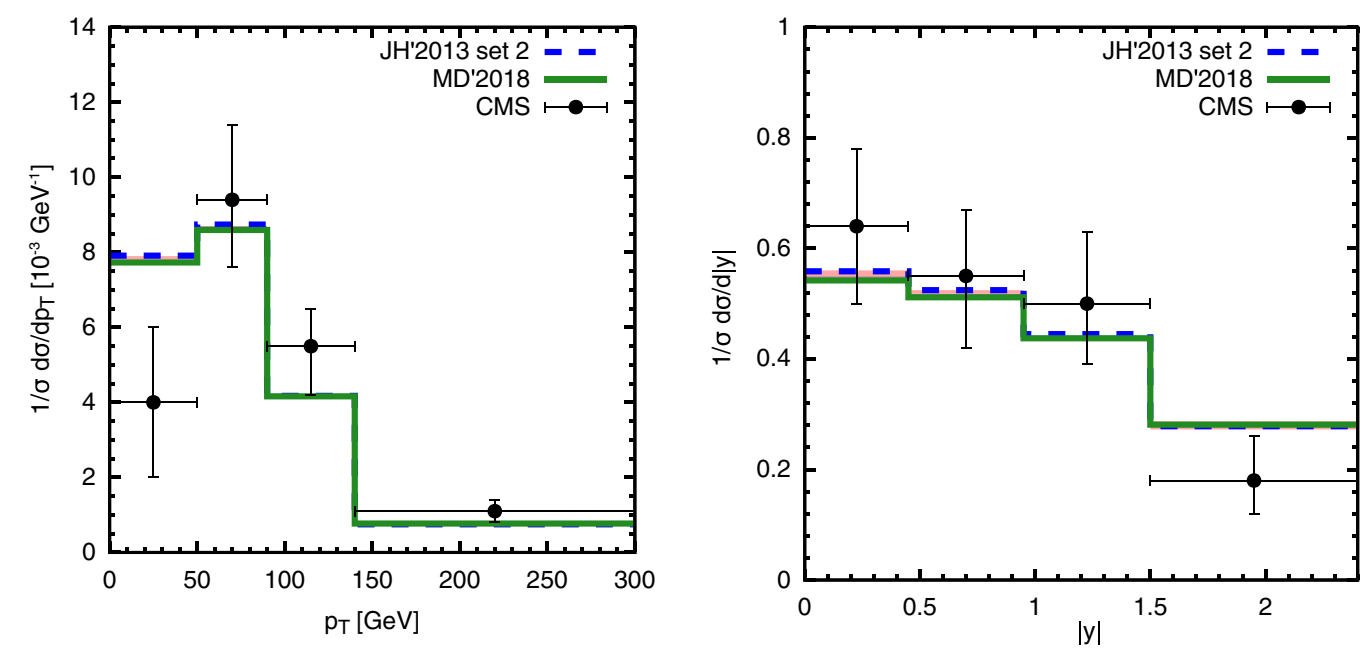

FIG. 8. The normalized differential cross sections of inclusive $t$-channel single top production at $\sqrt{s}=8 \mathrm{TeV}$ as functions of top quark transverse momentum and rapidity. Notation of the histograms is the same as in Fig. 2. The experimental data are from CMS [54].
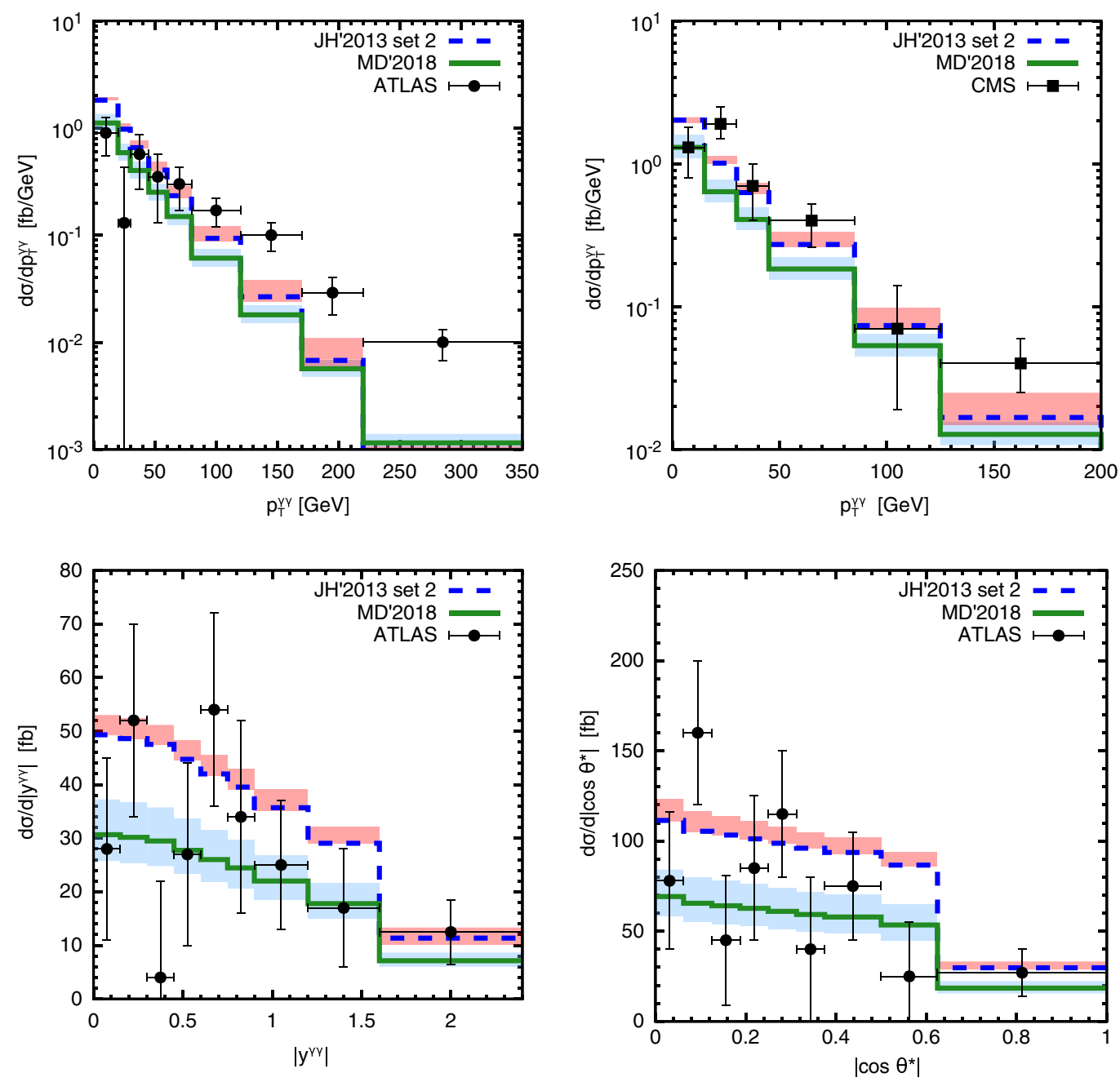

FIG. 9. The differential cross sections of inclusive Higgs boson production (in the diphoton decay mode) at $\sqrt{s}=13 \mathrm{TeV}$ as functions of diphoton pair transverse momentum $p_{T}^{\gamma \gamma}$, rapidity $\left|y^{\gamma \gamma}\right|$, and photon helicity angle $\cos \theta^{*}$ in the Collins-Soper frame. Notation of the histograms is the same as in Fig. 2. The experimental data are from CMS [62] and ATLAS [64]. 

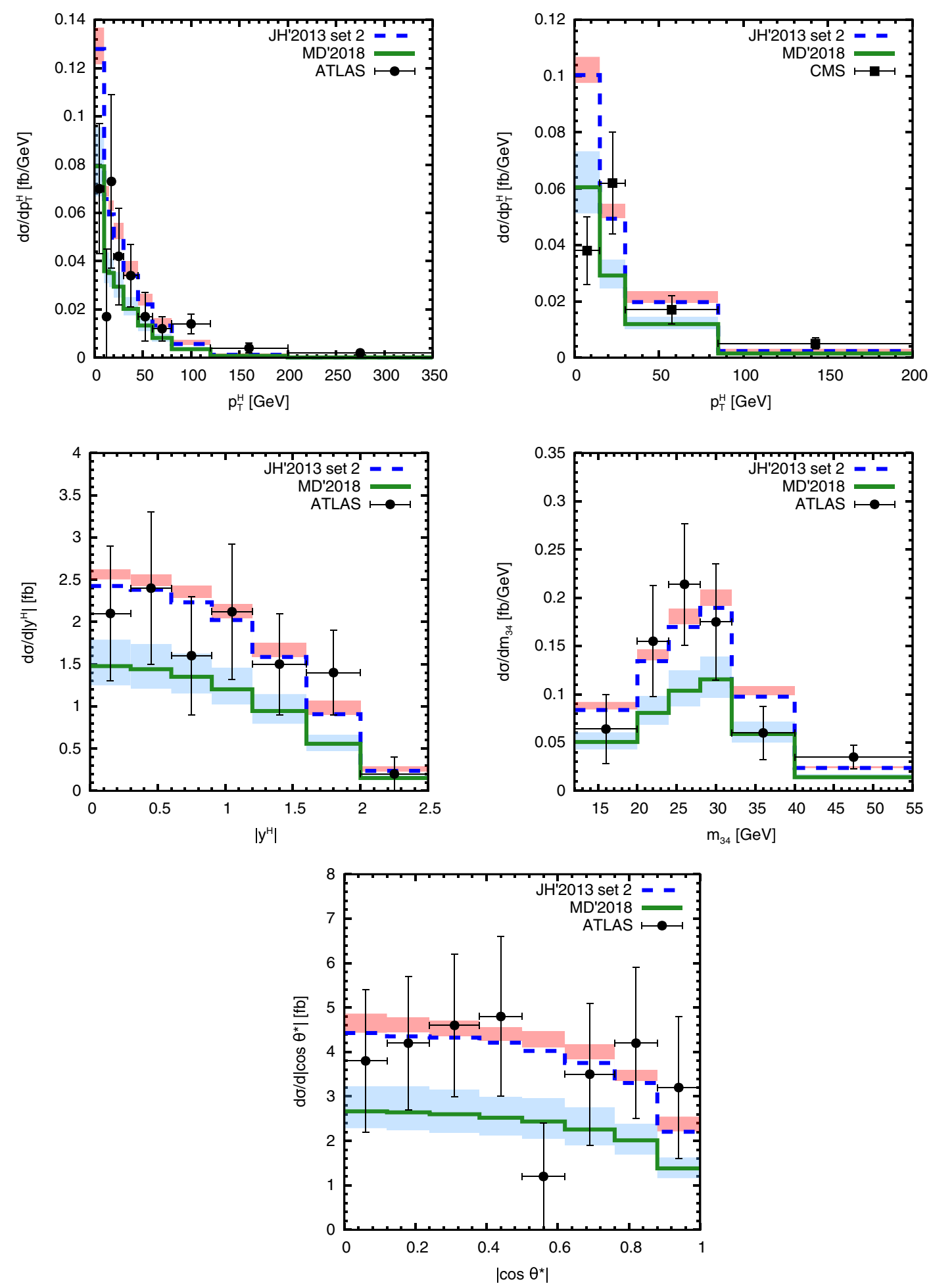

FIG. 10. The differential cross sections of inclusive Higgs production (in the $H \rightarrow Z Z^{*} \rightarrow 4 l$ decay mode) at $\sqrt{s}=13 \mathrm{TeV}$ as functions of Higgs boson transverse momentum $p_{T}^{H}$, rapidity $\left|y^{H}\right|$, leading lepton pair decay angle $\left|\cos \theta^{*}\right|$ (in the Collins-Soper frame), and invariant mass $m_{34}$ of the subleading lepton pair. Notation of the histograms is the same as in Fig. 2. The experimental data are from CMS [63] and ATLAS [65]. 
very sensitive to the TMD gluon density in a proton, in particular, to the parameters of the initial TMD gluon distribution. It could be important to further constrain the latter.

\section{CONCLUSION}

We have refined a fit of the experimental data on the inclusive spectra of the charged particles produced in the central $p p$ and $A A$ collisions at the RHIC and the LHC to determine the TMD gluon density in a proton at the starting scale. The parameters of this fit do not depend on the initial energy in a wide energy interval. Using a numerical solution of the CCFM gluon evolution equation, we extended the derived TMD gluon density (denoted as Moscow-Dubna 2018 or MD'2018 set) to a whole kinematical region and supplied it with the relevant TMD valence and sea quark distributions. The latter was calculated in the approximation where the gluon-to-quark splitting occurred at the last evolution step using the TMD gluon-to-quark splitting function. Some phenomenological applications of the proposed MD'2018 quark and gluon densities to the hard LHC processes were discussed. We achieved a good description of various data from HERA, RHIC, and LHC using the same set of parameters that confirms the link between soft processes at the LHC and low- $x$ physics at HERA, pointed out earlier. We demonstrated a significant influence of the initial nonperturbative gluon distribution on the description of the LHC data, which is important to further precise determination of the TMD quark and gluon densities in a proton.

\section{ACKNOWLEDGMENTS}

We would like to thank S. P. Baranov and F. Hautmann for very useful discussions and important remarks. A. V. L. and M.A.M. are grateful to the DESY Directorate for the support in the framework of the MoscowDESY project on Monte Carlo implementation for HERALHC. M. A. M. and N. A. A. were also supported by the Foundation for the Advancement of Theoretical Physics and Mathematics "Basis" (Grant Nos. 17-14-455-1 and 181-5-33-1). Part of this work was done by M. A. M. during his stay at DESY, funded by DAAD (program "Research Stays for University Academics and Scientists").
[1] R. Angeles-Martinez et al., Acta Phys. Pol. B 46, 2501 (2015).

[2] J. C. Collins, D. E. Soper, and G. F. Sterman, Nucl. Phys. B223, 381 (1983); B250, 199 (1985).

[3] S. Catani, M. Ciafaloni, and F. Hautmann, Nucl. Phys. B366, 135 (1991); J. C. Collins and R. K. Ellis, Nucl. Phys. B360, 3 (1991).

[4] L. V. Gribov, E. M. Levin, and M. G. Ryskin, Phys. Rep. 100, 1 (1983); E. M. Levin, M. G. Ryskin, Yu. M. Shabelsky, and A. G. Shuvaev, Sov. J. Nucl. Phys. 53, 657 (1991).

[5] E. Avsar, Int. J. Mod. Phys. Conf. Ser. 04, 74 (2011); arXiv:1203.1916.

[6] S. M. Aybat and T. C. Rogers, Phys. Rev. D 83, 114042 (2011).

[7] B. I. Ermolaev, M. Greco, and S. I. Troyan, Eur. Phys. J. C 71, 1750 (2011); 72, 1953 (2012).

[8] P. Kotko, K. Kutak, C. Marquet, E. Petreska, S. Sapeta, and A. Van Hameren, J. High Energy Phys. 09 (2015) 106.

[9] A. A. Grinyuk, A. V. Lipatov, G. I. Lykasov, and N. P. Zotov, Phys. Rev. D 93, 014035 (2016).

[10] A. V. Lipatov, G. I. Lykasov, and N. P. Zotov, Phys. Rev. D 89, 014001 (2014).

[11] A. A. Grinyuk, A. V. Lipatov, G. I. Lykasov, and N. P. Zotov, Phys. Rev. D 87, 074017 (2013).

[12] M. A. Kimber, A. D. Martin, and M. G. Ryskin, Phys. Rev. D 63, 114027 (2001); G. Watt, A. D. Martin, and M. G. Ryskin, Eur. Phys. J. C 31, 73 (2003).

[13] A. D. Martin, M. G. Ryskin, and G. Watt, Eur. Phys. J. C 66, 163 (2010).
[14] A. Bermudez Martinez, P. Connor, F. Hautmann, H. Jung, A. Lelek, V. Radescu, and R. Zlebcik, arXiv:1804.11152.

[15] V. N. Gribov and L. N. Lipatov, Sov. J. Nucl. Phys. 15, 438 (1972); L. N. Lipatov, Sov. J. Nucl. Phys. 20, 94 (1975); G. Altarelli and G. Parisi, Nucl. Phys. B126, 298 (1977); Yu. L. Dokshitzer, Sov. Phys. JETP 46, 641 (1977).

[16] F. Hautmann, H. Jung, A. Lelek, V. Radescu, and R. Zlebcik, Phys. Lett. B 772, 446 (2017).

[17] F. Hautmann, H. Jung, A. Lelek, and V. Radescu, J. High Energy Phys. 01 (2018) 070.

[18] F. Hautmann and H. Jung, Nucl. Phys. B883, 1 (2014).

[19] M. Ciafaloni, Nucl. Phys. B296, 49 (1988); S. Catani, F. Fiorani, and G. Marchesini, Phys. Lett. B 234, 339 (1990); Nucl. Phys. B336, 18 (1990); G. Marchesini, Nucl. Phys. B445, 49 (1995).

[20] A. B. Kaidalov, Z. Phys. C 12, 63 (1982); Surveys High Energy Phys. 13, 265 (1999); A. B. Kaidalov and O. I. Piskunova, Z. Phys. C 30, 145 (1986); Yad. Fiz. 43, 1545 (1986) [43, 994 (1986)].

[21] G. I. Lykasov and M. N. Sergeenko, Z. Phys. C 56, 697 (1992); 52, 635 (1991); 70, 455 (1996).

[22] V. A. Bednyakov, G. I. Lykasov, and V. V. Lyubushkin, Europhys. Lett. 92, 31001 (2010).

[23] http://tmd.hepforge.org.

[24] B. Andersson et al. (Small-x Collaboration), Eur. Phys. J. C 25, 77 (2002); J. Andersen et al. (Small-x Collaboration), Eur. Phys. J. C 35, 67 (2004); 48, 53 (2006).

[25] NA61 Collaboration, Eur. Phys. J. C 74, 2794 (2014).

[26] CMS Collaboration, Phys. Rev. Lett. 105, 022002 (2010). 
[27] ATLAS Collaboration, New J. Phys. 13, 053033 (2011).

[28] ALICE Collaboration, Eur. Phys. J. C 71, 1655 (2011).

[29] ALICE Collaboration, Phys. Lett. B 693, 53 (2010).

[30] ALICE Collaboration, Phys. Rev. D 82, 052001 (2010).

[31] ALICE Collaboration, Phys. Rev. D 88, 044910 (2013).

[32] STAR Collaboration, Phys. Rev. C 75, 064901 (2007).

[33] STAR Collaboration, Phys. Rev. Lett. 97, 152301 (2006).

[34] CMS Collaboration, Phys. Rev. D 97, 112003 (2018).

[35] F. Hautmann, H. Jung, and S. Taheri Monfared, Eur. Phys. J. C 74, 3082 (2014).

[36] E. A. Kuraev, L. N. Lipatov, and V. S. Fadin, Sov. Phys. JETP 44, 443 (1976); 45, 199 (1977); I. I. Balitsky and L. N. Lipatov, Sov. J. Nucl. Phys. 28, 822 (1978).

[37] K. Golec-Biernat and M. Wüsthoff, Phys. Rev. D 59, 014017 (1998); 60, 114023 (1999).

[38] Yu. V. Kovchegov, Phys. Rev. D 61, 074018 (2000).

[39] G. I. Lykasov and A. I. Malakhov, arXiv:1801.07250.

[40] A. V. Lipatov and N. P. Zotov, Phys. Lett. B 704, 189 (2011).

[41] D. A. Artemenkov, G. I. Lykasov, and A. I. Malakhov, Int. J. Mod. Phys. A 30, 1550127 (2015).

[42] V. Bednyakov, A. A. Grinyuk, G. I. Lykasov, and M. Poghosyan, Int. J. Mod. Phys. A 27, 1250042 (2012).

[43] H. Jung, S. P. Baranov, M. Deak, A. Grebenyuk, F. Hautmann, M. Hentschinski, A. Knutsson, M. Krämer, K. Kutak, A. V. Lipatov, and N. P. Zotov, Eur. Phys. J. C 70, 1237 (2010).

[44] J. Kwiecinski, A. D. Martin, and P. Sutton, Z. Phys. C 71, 585 (1996).

[45] B. Andersson, G. Gustafson, and J. Samuelsson, Nucl. Phys. B467, 443 (1996).

[46] F. Hautmann, M. Hentschinski, and H. Jung, Nucl. Phys. B865, 54 (2012).

[47] F. Hautmann, M. Hentschinski, and H. Jung, arXiv: 1207.6420 .

[48] S. Catani and F. Hautmann, Nucl. Phys. B427, 475 (1994); Phys. Lett. B 315, 157 (1993).

[49] G. P. Lepage, J. Comput. Phys. 27, 192 (1978).
[50] A. V. Kotikov, A. V. Lipatov, G. Parente, and N. P. Zotov, Eur. Phys. J. C 26, 51 (2002); A. V. Kotikov, A. V. Lipatov, and N. P. Zotov, Eur. Phys. J. C 27, 219 (2003).

[51] ZEUS Collaboration, J. High Energy Phys. 09 (2014) 127.

[52] H1 Collaboration, Eur. Phys. J. C 71, 1769 (2011); 72, 2252 (2012).

[53] H1 Collaboration, Eur. Phys. J. C 65, 89 (2010).

[54] CMS Collaboration, Report No. CMS-PAS-TOP-16-004, 2016.

[55] ATLAS Collaboration, Eur. Phys. J. C 77, 531 (2017).

[56] S. Alekhin, J. Blümlein, S. Moch, and R. Placakyte, Phys. Rev. D 94, 114038 (2016).

[57] Z. Sullivan, Europhys. J. Web Conf. 172, 03008 (2018).

[58] L. N. Lipatov and M. I. Vyazovsky, Nucl. Phys. B597, 399 (2001).

[59] A. V. Bogdan and V. S. Fadin, Nucl. Phys. B740, 36 (2006).

[60] PDG Collaboration, Chin. Phys. C 38, 090001 (2014).

[61] T. Stelzer, Z. Sullivan, and S. Willenbrock, Phys. Rev. D 56, 5919 (1997).

[62] CMS Collaboration, Report No. CMS-PAS-HIG-17-015, 2017.

[63] CMS Collaboration, Report No. RCMS-PAS-HIG-16-041, 2016.

[64] ATLAS Collaboration, arXiv:1802.04146.

[65] ATLAS Collaboration, J. High Energy Phys. 10 (2017) 132; Report No. ATLAS-CONF-2017-032, 2017.

[66] LHC Higgs Cross Section Working Group, arXiv:1101.0593.

[67] LHC Higgs Cross Section Working Group, arXiv:1201.3084.

[68] LHC Higgs Cross Section Working Group, arXiv:1307.1347.

[69] LHC Higgs Cross Section Working Group, arXiv:1610.07922.

[70] N. A. Abdulov, A. V. Lipatov, and M. A. Malyshev, Phys. Rev. D 97, 054017 (2018).

[71] J. R. Ellis, M. K. Gaillard, and D. V. Nanopoulos, Nucl. Phys. B106, 292 (1976).

[72] M. A. Shifman, A. I. Vainstein, M. B. Voloshin, and V. I. Zakharov, Sov. J. Nucl. Phys. 30, 711 (1979).

[73] A. V. Lipatov, M. A. Malyshev, and N. P. Zotov, Phys. Lett. B 735, 79 (2014).

[74] R. Islam, M. Kumar, and V. S. Rawoot, arXiv:1706.01402.

[75] A. Szczurek, M. Luszczak, and R. Maciula, Phys. Rev. D 90, 094023 (2014). 